\title{
ANÁLISIS DE CONFIABILIDAD Y DEL TIEMPO DE VIDA DE LAS \\ LÁMPARAS DEL PARQUE DE ALUMBRADO PÚBLICO DE LA ZONA NORTE DE LIMA METROPOLITANA
}

\section{Rosa Fátima Medina Merino}

\begin{abstract}
Resumen
Se realizó un estudio del tiempo de vida de las lámparas de alumbrado público de la zona norte de Lima metropolitana. Se consideraron cuatro tipos de lámparas suministradas por el distribuidor: Sodio 70W, Sodio 150W, Sodio 250W y Mercurio 125W. Se estableció como tiempo de observación un tiempo menor al ciclo teórico de 16000 horas señalado por el fabricante. Mediante el análisis exploratorio, para los datos del tiempo de vida, se detectaron valores discordantes para cada tipo de lámpara, y mediante pruebas de bondad de ajuste se determinó que los datos de la variable tiempo de vida provenían de una población con distribución de Weibull. Finalmente, se construyeron las tablas de vida para cada tipo de lámpara, y se realizaron las estimaciones del porcentaje de fallas para el parque de alumbrado público, mediante una aproximación a la distribución binomial.
\end{abstract}

Palabras clave: tiempo de vida, análisis exploratorio, valores discordantes, pruebas de bondad de ajuste, distribución Weibull, tablas de vida, distribución binomial

Analysis of reliability and bulbs lifespan of the public set lighting in the northern area of metropolitan Lima

\begin{abstract}
Summary
A study was conducted about the lifespan of the street lamps in the northern area of Metropolitan Lima, in which we considered four types of bulbs: Sodium 70W, Sodium 150W, Sodium 250W and Mercury 125W; with a shorter observation time than the theoretical cycle of 16000 hours stated by the manufacturer. Through the exploratory analysis of the data for the lifespan., discordant values were detected for each type of lamp, and through the goodness-of-fit tests it was determined that the above information came from a population with Weibull distribution. Finally, lifespan charts were created for each type of lamp, and the percentage of failures was estimated for the public set lighting, using an approximation to the Binomial distribution.
\end{abstract}

Key words: lifespan, exploratory analysis, discordant values, the goodness-of-fit tests,

Weibull distribution, lifespan charts, binomial distribution 


\section{Introducción}

El presente estudio muestra una aplicación estadística en el área de confiabilidad y tiempo de vida, la cual se enfoca en validar la estimación de lámparas apagadas por fallas que realiza la entidad responsable del suministro de energía eléctrica en la zona norte de Lima metropolitana. En este estudio se aplicaron herramientas estadísticas exploratorias, descriptivas e inferenciales.

El estudio surge a partir de la necesidad de la empresa distribuidora de energía eléctrica en la zona norte de Lima metropolitana, la misma que solicitó no mencionar su nombre, por demostrar que el porcentaje de fallas registradas en el parque de alumbrado eléctrico se deben a fallas en la calidad de las lámparas proporcionadas por el proveedor; de no demostrar esta situación se haría acreedora a una sanción por parte de la entidad supervisora. Por otro lado, es importante mencionar que al inicio del estudio se exigió una confidencialidad no menor a siete años contados a partir de la fecha de realización (2006), para hacer uso del informe con fines académicos, como es el caso de la presente publicación.

Inicialmente se realizó un reconocimiento de los datos proporcionados por la entidad responsable del suministro de energía eléctrica, en relación con los tiempos de falla (tiempo de vida) de las lámparas del parque de alumbrado público, y a partir de dicho reconocimiento se procedió a realizar las estimaciones tomando como horizonte las lámparas instaladas a partir del 1 enero de 2002 e intervenidas al 28 de mayo de 2005, con lo cual se tuvo un grupo de observación cuyas horas de vida aún no completan el ciclo teórico de 16000 horas señalado por el fabricante. Por esta misma razón no se realizaron tablas de vida ni estimaciones de proporción de falla para instalaciones más recientes.

\section{Objetivo y consideraciones del estudio}

\subsection{Objetivo general}

Estimar el porcentaje de lámparas del parque de alumbrado público de la zona norte de Lima metropolitana, que se encuentran apagadas debido a fallas.

\subsection{Consideraciones del estudio}

El estudio realizado presenta las siguientes consideraciones:

a) Cobertura geográfica. Parque de alumbrado público de la zona norte de Lima metropolitana.

b) Población en estudio. Lámparas del parque de alumbrado público. 
c) Unidad elemental. La unidad elemental es una lámpara del parque de alumbrado público, la cual puede ser de alguno de los siguientes cuatro tipos: Sodio 70W, Sodio 150W, Sodio 250W y Mercurio 125W.

\section{Reconocimiento de los datos}

\subsection{Descripción de los datos recabados}

Sea $X_{t}$ una variable aleatoria continua, definida como:

$X_{t}$ : Número de horas de vida de la lámpara instalada en el momento $t, t=1, \ldots, \mathrm{n}$

Se considera horas de vida a las horas transcurridas desde el día de la instalación de la lámpara hasta su fecha de reemplazo o hasta la última fecha de intervención registrada (28 de mayo del 2005). La realización de $X_{t}$ se da para $t^{3} 1$, es decir, para una o más lámparas instaladas en un mismo día.

\subsection{Preparación de la base de datos}

\subsubsection{Características de la base de datos}

La base de datos, con 8182 registros, fue proporcionada en el formato de archivo utilizado por Microsoft Access (MS Access). Esta base de datos fue exportada al MS Excel, y se encontraba compuesta principalmente por los siguientes campos:

Poste : Representa el código único que identifica al poste.

Fecha_imp : Fecha de instalación de la lámpara.

Fecha_Int : Fecha de intervención del poste (cambio de lámpara)

Tipo_Lam : Tipo de lámpara que ha sido implementado.

Días : Días transcurridos desde la instalación de la lámpara hasta su fecha de reemplazo o hasta la última fecha de intervención registrada.

Horas : Horas transcurridas desde la instalación de la lámpara hasta su fecha de reemplazo o hasta la última fecha de intervención registrada.

Entre otros campos que no son de interés para el presente artículo.

\subsubsection{Grupos de observación}

La variable de interés ha sido observada de acuerdo a cuatro tipos de lámparas; los tipos de lámpara y el porcentaje de lámparas por cada tipo se presentan en la tabla 1: 
Tabla 1. Número de lámparas instaladas según tipología

\begin{tabular}{|c|c|c|c|}
\hline Tipo de lámparas & Código & $\begin{array}{c}\text { No de lámparas } \\
\text { instaladas }\end{array}$ & $\begin{array}{c}\text { \% de lámparas } \\
\text { instaladas }\end{array}$ \\
\hline Mercurio $125 \mathrm{~W}$ & $\mathrm{H} 1$ & 2716 & 33.2 \\
\hline Sodio $70 \mathrm{~W}$ & S1 & 2748 & 33.6 \\
\hline Sodio $150 \mathrm{~W}$ & S2 & 1810 & 22.1 \\
\hline Sodio $250 \mathrm{~W}$ & S3 & 908 & 11.1 \\
\hline TOTAL & & 8182 & 100.0 \\
\hline
\end{tabular}

Elaboración propia.

Se realizó la verificación de diferencias entre los grupos de lámparas, es decir, se comprobó estadísticamente que el tiempo de vida, en horas, de las lámparas es diferente para cada tipo, tal como se aprecia en las tablas 2 y 3.

Tabla 2. Análisis de varianza para el tiempo de duración, en horas, de las lámparas

\begin{tabular}{|l|c|c|c|c|c|}
\hline & $\begin{array}{c}\text { Suma de } \\
\text { cuadrados }\end{array}$ & $\begin{array}{c}\text { Grados de } \\
\text { libertad }\end{array}$ & $\begin{array}{c}\text { Cuadrado } \\
\text { medios }\end{array}$ & F & P value \\
\hline Entre grupos & 889819041 & 5 & 177963808.2 & 13.067 & 0.000 \\
\hline $\begin{array}{l}\text { Dentro de los } \\
\text { grupos }\end{array}$ & 114099189944 & 8378 & 13618905.46 & & \\
\hline Total & 114989008985 & 8383 & & & \\
\hline
\end{tabular}

Elaboración propia.

Tabla 3. Comparaciones entre tipos de lámparas

\begin{tabular}{|c|c|c|}
\hline \multicolumn{3}{|c|}{ Variable dependiente: Horas LSD } \\
\hline (I) Tipo 1 & (J) Tipo 1 & P_value \\
\hline H1 & S1 & 0.705 \\
\hline & S2 & 0.000 \\
\hline & S3 & 0.000 \\
\hline S1 & S2 & 0.000 \\
\hline & S3 & 0.001 \\
\hline S2 & S3 & 0.103 \\
\hline
\end{tabular}

Elaboración propia. 
Al realizar las comparaciones del tiempo de funcionamiento por cada par de tipo de lámparas, se observa lo siguiente:

- No existen diferencias significativas de los tiempos de vida entre los siguientes tipos de lámparas: H1 y S1, S2 y S3.

- Existe una diferencia significativa de los tiempos de vida entre los siguientes tipos de lámparas: H1 y S2, H1 y S3, S1 y S2, S1 y S3.

\section{Análisis preliminar}

\subsection{Análisis exploratorio}

Antes de la determinación del porcentaje de fallas se realizó el análisis exploratorio del número de horas de vida $\left(X_{t}\right)$, según los tipos de lámparas, con la siguiente finalidad:

a) Identificar los valores discordantes (outliers) de la variable, y sus puntos de corte asociados.

b) Explorar la simetría de la variable.

c) Explorar el tipo de distribución.

El análisis exploratorio se realizó mediante el diagrama de cajas para cada tipo de lámpara (ver figura 1) en la cual se observa que en cada tipo de lámparas existe un porcentaje considerable de valores discordantes inferiores, los cuales distorsionarían el tipo de distribución a ajustar. En la tabla 4 se presenta el número y el porcentaje que representan las lámparas con valores discordantes.

Figura 1. Diagrama de cajas por tipo de lámpara

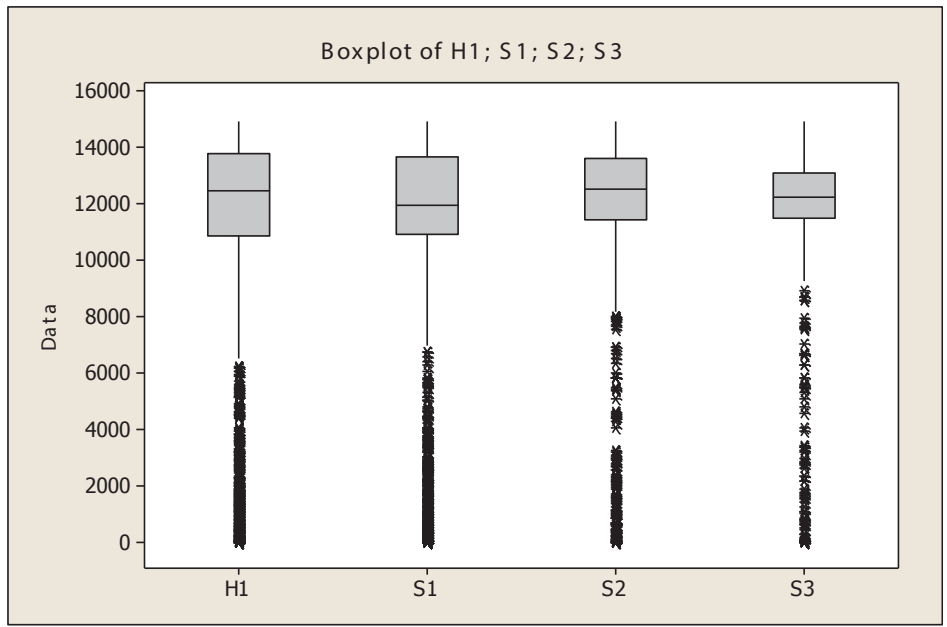

Elaboración propia. Gráfica del software Minitab. 
Tabla 4. Cantidad y porcentaje de datos outliers según tipo de lámpara

\begin{tabular}{|c|c|c|c|c|}
\hline $\begin{array}{c}\text { Tipo de } \\
\text { lámpara }\end{array}$ & $\begin{array}{c}\text { Cantidad de } \\
\text { lámparas }\end{array}$ & $\begin{array}{c}\text { Punto de corte } \\
\text { inferior }\end{array}$ & $\begin{array}{c}\text { Cantidad de lámparas } \\
\text { con valores outliers* }\end{array}$ & $\begin{array}{c}\% \text { de } \\
\text { lámparas }\end{array}$ \\
\hline H1 & 2716 & 6486.0 & 381 & 14.0 \\
\hline S1 & 2748 & 6787.5 & 349 & 12.7 \\
\hline S2 & 1810 & 8112.0 & 139 & 7.7 \\
\hline S3 & 908 & 9214.5 & 87 & 9.6 \\
\hline
\end{tabular}

* Tiempo de funcionamiento menor al punto de corte inferior.

Elaboración propia.

Los valores outliers representan el $11.7 \%$ del total de las observaciones, y no fueron considerados en la determinación de la función de distribución de los tipos de lámparas. Es importante señalar que estos valores indican que existe un grupo de lámparas, para cada tipo, cuya duración es inusualmente menor a lo garantizado por el fabricante; este resultado debería ser considerado por la empresa distribuidora de energía eléctrica de Lima norte como salvaguarda ante la entidad fiscalizadora de la calidad del servicio que se presta a los usuarios. En la figura 2 se muestra el diagrama de tallos y hojas correspondiente a las lámparas de mercurio de $125 \mathrm{~W}(\mathrm{H} 1)$.

Figura 2. Diagrama de tallos y hojas del número de horas de funcionamiento de las lámparas H1

\begin{tabular}{|c|c|c|}
\hline \multicolumn{3}{|c|}{$\begin{array}{l}\text { Lámparas de Mercurio } 125 \mathrm{~W}-\mathrm{H} 1 \\
\mathrm{~N}=2716 \\
\text { Leaf Unit }=100\end{array}$} \\
\hline 152 & 0 & $000000000000000000000000000000000001111111111111222222222+$ \\
\hline 246 & 1 & $000000111111111112222222222233333333333344444444455555566+$ \\
\hline 296 & 2 & 00000001111111122223333333455556667777777888999999 \\
\hline 333 & 3 & 0001111222222233344455666677778888899 \\
\hline 354 & 4 & 001444555555666788899 \\
\hline 375 & 5 & 111112233344555678899 \\
\hline 389 & 6 & 00122256779999 \\
\hline 407 & 7 & 001244455677777778 \\
\hline 430 & 8 & 00123334445555666777799 \\
\hline 450 & 9 & 00011233356667778899 \\
\hline 767 & 10 & $224445555555555555555555555555555555555555555566666666666+$ \\
\hline 1338 & 11 & $000000000000000000000000000000000000000000000000000000011+$ \\
\hline$(265)$ & 12 & $000001111222223333344444444444445555555555555555555555555+$ \\
\hline 1113 & 13 & $000000000000000000000000011111111111111111111111111111111+$ \\
\hline 538 & 14 & $000000000000000000000000000000000000000000000001111111111+$ \\
\hline
\end{tabular}

Elaboración propia. Reporte del software Minitab.

En las lámparas tipo H1 se observa que el número de horas de funcionamiento se distribuye en forma asimétrica y su configuración bimodal, lo cual es característico 
de la distribución tipo bañera, según lo señalan Meza (1994) y Meyer (1990). Similar situación se presenta en los demás tipos de lámparas.

En la tabla 5 se presentan los resultados del análisis descriptivo, en relación con los indicadores de centralidad y de dispersión, y se observa que las lámparas cuya mayoría de tiempos de funcionamiento son superiores a la mediana general, 12 500 horas, se comportan en forma más homogénea que aquellas lámparas que tienen un tiempo de funcionamiento inferior a la mediana general; como es el caso de las lámparas de sodio 150 W (S2).

Tabla 5. Estadísticas descriptivas del número de horas de funcionamiento según tipo de lámpara

\begin{tabular}{|l|c|c|c|c|}
\hline & $\mathrm{H} 1$ & $\mathrm{~S} 1$ & $\mathrm{~S} 2$ & $\mathrm{S3}$ \\
\hline No de lámparas & 2716 & 2748 & 1810 & 908 \\
\hline Promedio & 11125.12 & 11162.90 & 11866.76 & 11622.25 \\
\hline Mediana & 12450.00 & 11952.00 & 12516.00 & 12252.00 \\
\hline Desviación estándar & 4072.808 & 3922.116 & 3101.854 & 3060.421 \\
\hline Mínimo & 12 & 12 & 12 & 12 \\
\hline Máximo & 14916 & 14916 & 14904 & 14904 \\
\hline Coeficiente de variación & $36.6 \%$ & $35.1 \%$ & $26.1 \%$ & $26.3 \%$ \\
\hline
\end{tabular}

Elaboración propia.

En la tabla 6 se presenta una tabla cruzada en base a las variables tipo de lámpara (Tipo_Lam) y el año de intervención. Esta última variable se determinó en base a la fecha de intervención (Fecha_Int), en comparación con su fecha de instalación (Fecha_imp).

Tabla 6. Número de lámparas reemplazadas según el año de intervención

\begin{tabular}{|c|c|c|c|c|c|}
\hline \multirow{2}{*}{$\begin{array}{l}\text { Tipo de } \\
\text { lámpara }\end{array}$} & \multicolumn{4}{|c|}{ Año de intervención } & \multirow[b]{2}{*}{ Total } \\
\hline & $\begin{array}{c}\text { Antes del } \\
\text { primer año }\end{array}$ & $\begin{array}{c}\text { Entre el primero y } \\
\text { el segundo año }\end{array}$ & $\begin{array}{c}\text { Entre el segundo } \\
\text { y el tercer año }\end{array}$ & $\begin{array}{c}\text { Al tercer año } \\
\text { o más }\end{array}$ & \\
\hline $\mathrm{H} 1$ & 336 & 90 & 1222 & 1068 & 2716 \\
\hline S1 & 316 & 69 & 1369 & 994 & 2748 \\
\hline S2 & 108 & 39 & 1010 & 653 & 1810 \\
\hline S3 & 57 & 29 & 611 & 211 & 908 \\
\hline Total & 817 & 227 & 4212 & 2926 & 8182 \\
\hline
\end{tabular}

Elaboración propia. 
La representación gráfica de la tabla 6, en base a los porcentajes que representa cada año de intervención para cada tipo de lámpara, se presenta en la figura 3.

Figura 3. Porcentaje de lámparas reemplazadas según el año de intervención

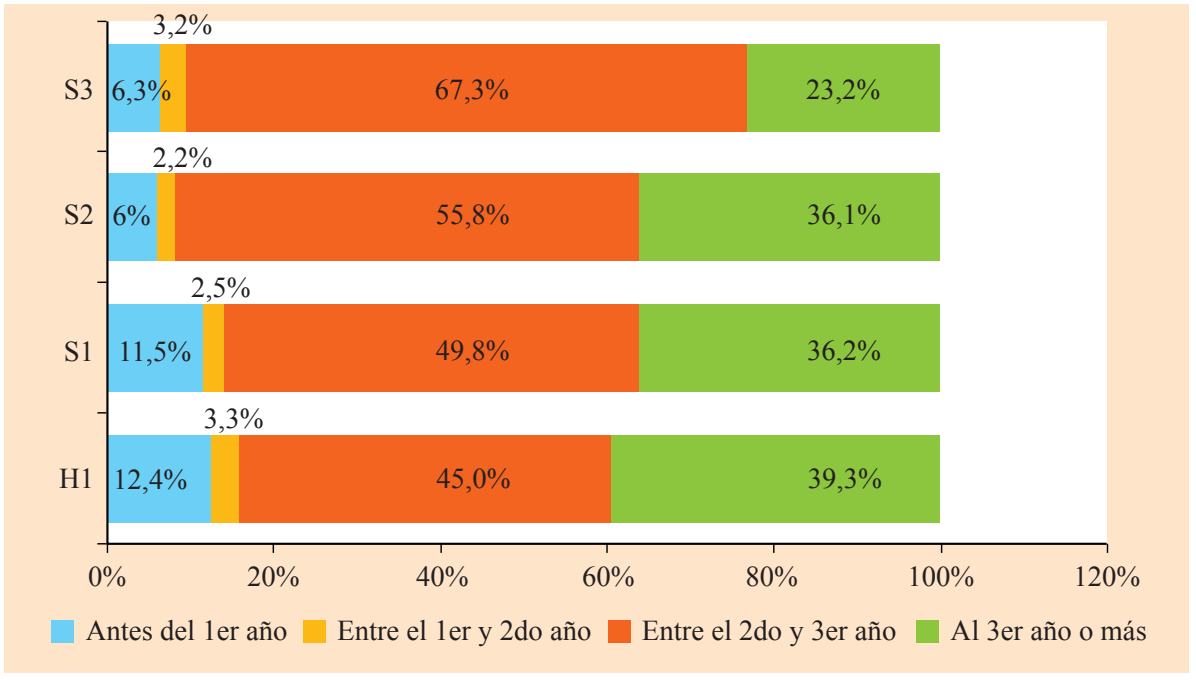

Elaboración propia.

\subsection{Análisis del tiempo de vida de las lámparas}

El enfoque inferencial de la estadística permite analizar el comportamiento de los datos obtenidos de una muestra, y a partir de ella extraer conclusiones acerca de la población de la cual se obtuvo la muestra. Considerando que se está trabajando con una muestra total de 8182 lámparas, se usaron métodos paramétricos con la finalidad de determinar el tipo de distribución de la variable, hallar la probabilidad de falla en forma puntual e interválica, y determinar las tablas de vida y confiabilidad para la distribución encontrada.

\subsubsection{Conceptos básicos}

\section{a) Tiempo de falla}

El tiempo de falla se constituye a partir de los siguientes tres elementos:

Tiempo inicial.- El tiempo de inicio del estudio debe estar definido claramente, y los individuos deben ser comparables en el origen del estudio, con excepción de las diferencias medidas por las covariables.

Escala de medida.- Usualmente se considera como escala al tiempo real, a 
pesar de existir otras alternativas; en pruebas de ingeniería se usan otras escalas de medida, como el número de ciclos, el kilometraje de un vehículo, entre otras.

Evento de interés (falla).- Es importante, en estudios de sobrevivencia, definir de forma clara y precisa lo que viene a ser una falla; en algunas situaciones, la definición de falla ya es clara, y en otras puede asumir términos ambiguos; por ejemplo, un producto se considerará inapropiado para el consumo cuando contiene más de una determinada concentración de microorganismos por milímetro cuadrado $\left(\mathrm{mm}^{2}\right)$ de área del producto.

\section{b) Función de sobrevivencia}

La función de sobrevivencia, tal como señalan Colosimo et al. (2002), está definida como la probabilidad de que una observación no falle antes de un tiempo t. En términos probabilísticos, se define como:

$$
S(t)=P(T \geq t)
$$

\section{c) Función de la tasa de falla}

La probabilidad de falla ocurre en un intervalo de tiempo $\left[t_{1}, t_{2}\right\rangle$ y puede ser expresada en términos de la función de sobrevivencia como:

$$
S\left(t_{1}\right)-S\left(t_{2}\right)
$$

La tasa de falla en el intervalo $\left[t_{1}, t_{2}\right\rangle$ está definida como la probabilidad de que una falla ocurra en este intervalo, dado que no ocurre antes de $t_{1^{\prime}}$ y se expresa por:

$$
\frac{S\left(t_{1}\right)-S\left(t_{2}\right)}{\left(t_{2}-t_{1}\right) S\left(t_{1}\right)}
$$

Redefiniendo el intervalo como $\left[t_{1}, t_{1}+\Delta t\right\rangle$, y en forma general como $[t, t+\Delta t\rangle$, se llega a la siguiente expresión:

$$
h(t)=\frac{S(t)-S(t+\Delta t)}{\Delta t S(t)}
$$

La expresión $h(t)$ representa la tasa de falla instantánea en el tiempo $t$, condicional a la sobrevivencia antes del tiempo $t$. Una función de tasa de falla de $T$ es entonces definida como:

$$
h(t)=\lim _{\Delta t \rightarrow 0} \frac{P(t \leq T<t+\Delta t \mid T \geq t)}{\Delta t}
$$

La figura 4 muestra tres funciones de tasa de falla. La función 1 es creciente, la 2 es constante y la 3 es decreciente. La función creciente indica que la tasa de falla aumenta con el transcurrir del tiempo; este comportamiento muestra un efecto 
gradual del desgaste. La función constante indica que la tasa de falla no se altera con el pasar el tiempo. La curva decreciente muestra que la tasa de falla disminuye a medida que el tiempo pasa. La combinación de las curvas 1, 2 y 3 es conocida como "Curva de la bañera", la cual tiene una tasa de falla decreciente en el período inicial, constante en la fase intermedia y creciente en el período final.

Figura 4. Funciones de tiempo de falla

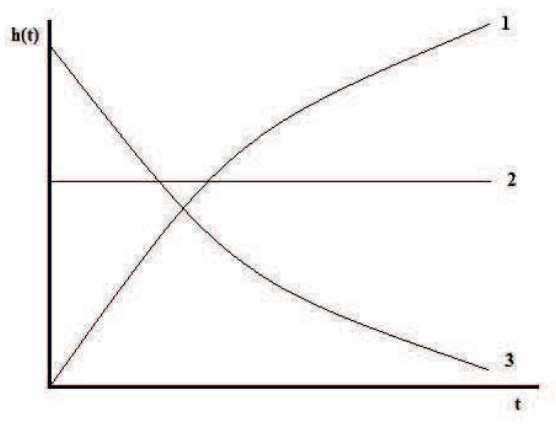

Elaboración propia.

Existe una relación entre la función de tasa de falla $h(t)$ y la función de sobrevivencia $S(t)$. Se tiene que:

$$
h(t)=\frac{f(t)}{S(t)}
$$

donde $f(t)$ es la función de densidad de T. Otra relación muy usada es la que relaciona la función de sobrevivencia con la función de tasa de falla acumulada $H(t)$, es decir:

$$
H(t)=\int_{0}^{t} h(u) d u=-\log (S(t))
$$

\section{d) Vida media residual}

Un indicador de interés en el análisis de sobrevivencia es la vida media residual (Freitas y Colosimo, 1997), la cual es definida como condicional a un cierto tiempo de vida $t$, es decir, para individuos con edad t, esta cantidad mide el tiempo medio restante de vida. La vida media residual es entonces el área sobre la curva de sobrevivencia en dirección del tiempo t dividida por S(t). Esto es:

$$
v m r(t)=\frac{\int_{t}^{\infty}(u-t) f(u) d u}{S(t)}=\frac{\int_{t}^{\infty} S(u) d u}{S(t)}
$$




\subsubsection{Ajuste de la distribución}

Para el caso de nuestro interés, se probó el ajuste de los tiempos de falla a una distribución Weibull, la cual es muy usada en los análisis de confiabilidad, ya que se trata de una distribución muy flexible, mediante la que se pueden modelar las tres etapas típicas de la curva de la bañera, y que salió a relucir mediante el análisis exploratorio. Estas tres etapas corresponden a:

a) Etapa inicial, con la cual se presenta una tasa creciente de fallas o defectos.

b) Etapa de vida útil, en esta etapa la tasa de falla es aproximadamente estable

c) Etapa de finalización, en la cual la tasa de falla es creciente.

Para la realización de la prueba de bondad de ajuste se plantean las siguientes hipótesis para cada tipo de lámpara:

H0: Tiempos de falla de cierto tipo de lámpara se distribuyen como una Weibull

H1: Tiempos de falla de cierto tipo de lámpara no se distribuyen como una Weibull

Para la verificación se utilizó la prueba de Anderson y los métodos gráficos de probabilidad por contraste. La decisión se obtuvo por comparación con el valor P-value para un nivel de significancia de 0.01. En la figura 5 se muestra el resultado del contraste para la lámpara de mercurio de $125 \mathrm{~W}(\mathrm{H} 1)$.

Figura 5. Gráfico de probabilidad de contraste con distribución Weibull para lámpara H1

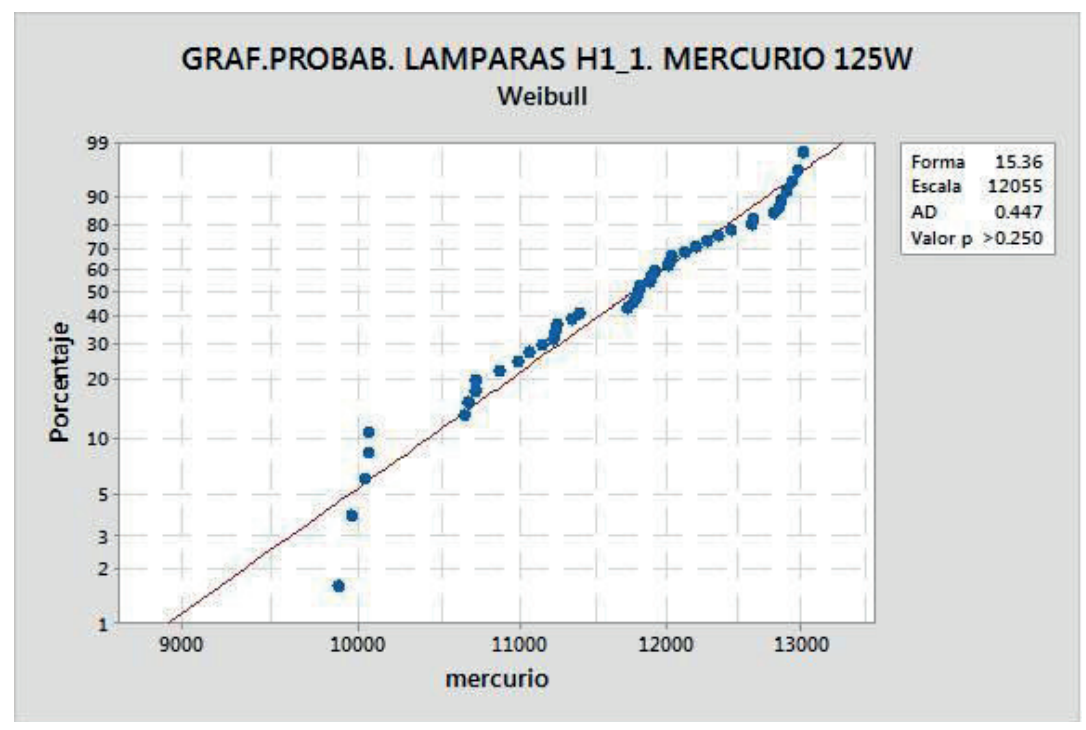

Elaboración propia. Gráfica del software Minitab. 
Para la lámpara H1, como para los demás tipos de lámparas, se verifica que cada una de estas curvas sigue la distribución Weibull, ya que los P value obtenidos son mayores al valor de significancia fijado (0.05), cuyos parámetros se muestran en la tabla 7.

Conocida la distribución, se construyeron las tablas de vida y confiabilidad que permitirán estimar la tasa de falla para cada día, el tiempo esperado de duración de la lámpara y el número esperado de lámparas que fallen en el parque de alumbrado público, bajo el supuesto de una instalación en el día en estudio.

Tabla 7. Parámetros estimados de la distribución Weibull por tipo de lámpara

\begin{tabular}{|c|c|c|}
\hline \multirow{2}{*}{ Tipo de lámpara } & \multicolumn{2}{|c|}{ Parámetros de la distribución Weibull } \\
\cline { 2 - 3 } & $\alpha$ & $\beta$ \\
\hline Mercurio $125 \mathrm{~W}$ & 15.36 & 12055 \\
\hline Sodio $70 \mathrm{~W}$ & 21.32 & 12162 \\
\hline Sodio $150 \mathrm{~W}$ & 12.06 & 11655 \\
\hline Sodio $250 \mathrm{~W}$ & 11.90 & 11764 \\
\hline
\end{tabular}

Elaboración propia.

\section{Análisis del tiempo de vida de las lámparas instaladas}

\subsection{Metodología para la obtención de tablas de vida}

La elaboración de una tabla de vida, de acuerdo con Vallenas (2000), consiste en dividir el tiempo en cierto número de intervalos; por lo tanto, se dividirá en $k+1$ intervalos, definidos por los siguientes puntos de corte: $t_{1}, t_{2}, \ldots, t_{k}$. Esto es, $I_{\mathrm{i}}=\left[t_{\mathrm{i}-1}, t_{\mathrm{i}}\right\rangle ; \mathrm{i}=1, \ldots, k+1$, donde $t_{0}=0$ y $t_{\mathrm{k}+1}=+\infty$.

Sea $q_{\mathrm{i}}$ : probabilidad de que una lámpara falle dentro del intervalo $\left[t_{\mathrm{i}-1}, t_{\mathrm{i}}\right\rangle$, si es que no falla antes de $t_{\mathrm{i}-1}$. La estimación de $q_{\mathrm{i}}$ en la tabla de vida está dada por:

$$
\hat{q}_{i}=\frac{\text { Número de fallas en el intervalo }\left[t_{i-1}, t_{i}\right\rangle}{\text { Número en riesgo en } t_{i-1}-\left(\text { Número de censuras en }\left[t_{i-1}, t_{i}\right\rangle\right) / 2}
$$

Se asume que existen $n$ observaciones en el estudio y $k$ fallas $(k \leq n)$ distintas en los tiempos $t_{1}<t_{2}<\ldots<t_{\mathrm{k}}$. Asimismo, $S(t)$ se considera como una función discreta con probabilidad mayor que cero en los tiempos de falla $t_{\mathrm{i}}, i=1, \ldots, k$. Por lo tanto, el estimador de la tabla de vida es:

$$
\hat{S}(t)=\left(1-q_{1}\right)\left(1-q_{2}\right) \ldots\left(1-q_{i}\right)
$$




\subsection{Estimación probabilística del tiempo de falla}

Una vez identificado el tipo de distribución se procedió a estimar, de manera puntual y por intervalos, el tiempo de falla de las lámparas. La variable aleatoria $X$, que cuenta el número de éxitos en los $n$ ensayos, tiene una distribución binomial con parámetros n y p, cuya función de probabilidades, según la definen Meza (1994) y Mood y Graybill (1976) está dada por:

$$
f(x)=P(X=x)=\left(\begin{array}{l}
n \\
x
\end{array}\right) \pi^{x}(1-\pi)^{n-x}, \mathrm{x}=0,1,2, \ldots, \mathrm{n}
$$

en donde:

- Cada ensayo tiene solo dos resultados posibles: "defectuosa"y "no defectuosa".

- El éxito corresponde al resultado de lámpara defectuosa; la probabilidad de éxito en cada ensayo, denotada por p, permanece constante.

Así, se tienen los siguientes resultados:

Tabla 8. Cantidad y proporción de lámparas defectuosas según tipo

\begin{tabular}{|c|c|c|}
\hline \multirow{2}{*}{ Tipo de lámpara } & \multicolumn{2}{|c|}{ Lámparas defectuosas } \\
\cline { 2 - 3 } & Cantidad & Proporción \\
\hline Mercurio $125 \mathrm{~W}$ & 101 & 0.0433 \\
\hline Sodio $70 \mathrm{~W}$ & 124 & 0.0517 \\
\hline Sodio $150 \mathrm{~W}$ & 45 & 0.0269 \\
\hline Sodio $250 \mathrm{~W}$ & 19 & 0.0231 \\
\hline Total & 297 & 0.0400 \\
\hline
\end{tabular}

Elaboración propia.

Tabla 9. Límites de confianza para la proporción de defectuosos según tipo de lámpara

\begin{tabular}{|c|c|c|c|c|c|}
\hline & $\mathrm{H} 1$ & $\mathrm{~S} 1$ & $\mathrm{~S} 2$ & $\mathrm{~S} 3$ & TOTAL \\
\hline Número de fallas & 101 & 124 & 45 & 19 & 297 \\
\hline Proporción de fallas $=p$ & 0.0433 & 0.0517 & 0.0269 & 0.0231 & 0.04 \\
\hline$p^{*} q$ & 0.0414 & 0.0490 & 0.0262 & 0.0226 & 0.0384 \\
\hline Raíz (n) & 10.049 & 11.1355 & 6.7082 & 4.3589 & 17.2337 \\
\hline varianza & 0.0041 & 0.0044 & 0.0039 & 0.0052 & 0.0022 \\
\hline Varianza *1.96 & 0.0081 & 0.0086 & 0.0076 & 0.0101 & 0.0044 \\
\hline Límite inferior & 0.0352 & 0.0431 & 0.0193 & 0.0129 & 0.0356 \\
\hline Límite superior & 0.0514 & 0.0603 & 0.0345 & 0.0332 & 0.0444 \\
\hline
\end{tabular}

Elaboración propia. 
Tabla 10. Equivalente de lámparas defectuosas según tipo de lámpara

\begin{tabular}{|c|c|c|c|c|c|}
\hline & $\mathrm{H} 1$ & $\mathrm{~S} 1$ & $\mathrm{~S} 2$ & $\mathrm{~S} 3$ & TOTAL \\
\hline Número de fallas & 1777 & 8408 & 1610 & 560 & 11511 \\
\hline Proporción de fallas $=p$ & 0.0433 & 0.0517 & 0.0269 & 0.0231 & 0.04 \\
\hline Límite inferior & 0.0352 & 0.0431 & 0.0193 & 0.0129 & 0.0356 \\
\hline & 1461 & 7009 & 1155 & 313 & 10245 \\
\hline Límite superior & 0.0514 & 0.0603 & 0.0345 & 0.0332 & 0.0444 \\
\hline & 2110 & 9806 & 2065 & 805 & 12778 \\
\hline
\end{tabular}

Elaboración propia.

\section{Conclusiones y recomendaciones}

\subsection{Conclusiones}

1. Se determinó la existencia de un alto porcentaje de lámparas que fallan a muy corto plazo, y que originan datos outliers; el caso más notorio es el correspondiente a las lámparas de mercurio $125 \mathrm{~W}$.

2. El análisis exploratorio de datos permitió determinar que el tiempo de vida de las lámparas presenta la distribución del tipo bañera, la cual tiene una tasa de falla decreciente en el período inicial, constante en la fase intermedia y creciente en el período final; cada fase puede ser modelada mediante una distribución de weibull.

3. La estimación del porcentaje de fallas para los tipos de lámparas permitió una estimación adecuada de las posibles fallas para lámparas instaladas entre enero de 2002 y mayo de 2003.

4. En general, el porcentaje de fallas para el parque de alumbrado público es de $4 \%$, lo que equivale a una estimación de 297 posibles fallas para lámparas instaladas entre enero de 2002 y mayo de 2003.

\subsection{Recomendaciones}

Los resultados obtenidos mediante este estudio, a partir de una cohorte no completa para el periodo de vida sugerido por el fabricante, 16000 horas, tienen un carácter de aproximación al 81.25 \%, en tal sentido se recomienda lo siguiente:

- Completar una cohorte para determinar al 100 \% una estimación del porcentaje de fallas del parque. 
- Completar 3 o más cohortes con la finalidad de elaborar un modelo mediante tablas de vida para estimaciones de tasas de fallas, tiempo medio de duración y sobrevivencia máxima.

- También es importante para lo que corresponde a la base de datos determinar con mayor rigurosidad la fecha exacta de instalación y la fecha de observación.

\section{Referencias}

Besterfield, D. H. (2004). Control de calidad. México, D. F., México: Prentice Hall Hispanoamericana.

Colosimo, E., Ferreira, F., Oliveira, M., \& Souza, C. (2002). Empirical comparisons between Kaplan-Meier and survival function estimators. Journal of Statistical Computation and Simulation, 4(72), 299-308.

Freitas, M., \& Colosimo, E. (1997). Confiabilidade: análise de tempo de falha e testes de vida. Belo Horizonte, Brasil: Fundação Christiano Ottoni.

Meyer, P. (1990). Introductory probability and statistical applications. Massachusetts, Estados Unidos: Addison Wesley.

Meza, E. (1994). Probabilidad. Lima, Perú: Editores A\&B.

Mood, A. \& Graybill, F. (1976). Introducción a la teoría de la estadística. Madrid, España: Aguilar S. A Ediciones.

Vallenas, G. (2000). Indicadores demográficos. Lima, Perú: INEI.

\section{GLOSARIO}

- Análisis de sobrevivencia. Se denomina análisis de sobrevivencia (análisis de supervivencia) al conjunto de técnicas que permiten estudiar la variable tiempo hasta que ocurre un evento y su dependencia de otras posibles variables explicatorias.

- Análisis de varianza. Técnica estadística con que se prueba la igualdad de 3 o más medias muestrales y que, por tanto, permite hacer inferencias sobre si las muestras provienen de poblaciones que tienen la misma media.

- Confiabilidad. Es la probabilidad de que un producto desempeñe la función para la que fue creado, durante una vida determinada y bajo ciertas condiciones definidas. 
- Distribución Weibull. Es la distribución de probabilidad que describe los tiempos de vida T para un producto fabricado. Si la variable $T$ sigue una distribución Weibull, su función de probabilidad está representada como

$$
f_{T}(t)=\alpha \beta t^{\beta-1} e^{-\alpha t^{\beta}} .
$$

- Método paramétrico. Conjunto de técnicas estadísticas que hacen suposiciones restrictivas sobre la forma de una distribución de población, cuando se realiza una prueba de hipótesis.

- Outlier. Conocido también como valor atípico, discordante o aberrante. Valor de una observación que tiene un comportamiento distinto a las demás.

- Parámetro. Valor que describe el comportamiento de una población.

- Tablas de vida. Presentación ordenada cronológicamente de un grupo de individuos a los cuales se les hace un seguimiento desde su origen hasta su muerte o desaparición, registrando como variable de interés el número de sobrevivientes en el tiempo y la probabilidad de sobrevivencia.

- Tasa de falla. Es la probabilidad de que una unidad de producto falle durante una unidad específica de tiempo o de ciclos. 